\title{
Critical Points of Solutions of Degenerate Elliptic Equations in the Plane
}

\author{
Simone Cecchini ${ }^{* \dagger} \quad$ Rolando Magnanini ${ }^{\ddagger}$
}

November 9, 2018

\begin{abstract}
We study the minimizer $u$ of a convex functional in the plane which is not Gâteaux-differentiable. Namely, we show that the set of critical points of any $C^{1}$-smooth minimizer can not have isolated points. Also, by means of some appropriate approximating scheme and viscosity solutions, we determine an Euler-Lagrange equation that $u$ must satisfy. By applying the same approximating scheme, we can pair $u$ with a function $v$ which may be regarded as the stream function of $u$ in a suitable generalized sense.

Keywords: Quasi-linear degenerate elliptic partial differential equations, critical points of solutions, stream functions, non-differentiable variational functional. MSC: 35B05,35B38,35J20,35J60.
\end{abstract}

\section{Introduction}

\subsection{Motivations: a case study.}

This paper will mainly focus on the properties of certain convex coercive nondifferentiable functionals and their extremals. We are partly motivated by the investigations that the second author and G. Talenti pursued in a series of papers [18]-20] about complex-valued solutions of the classical eikonal equation in the plane.

One of the main charachters acting in those papers is the functional

$$
\mathcal{J}(u)=\int_{\Omega} f(|\nabla u|) d x,
$$

where $\Omega$ is a bounded domain in $\mathbb{R}^{N}$ (therein, with $N=2$ ), $|\nabla u|$ denotes the modulus of the gradient of a scalar function $u$ defined in $\Omega$ and

$$
f(\rho)=\frac{1}{2}\left[\rho \sqrt{1+\rho^{2}}+\log \left(\rho+\sqrt{1+\rho^{2}}\right)\right] \text { for } \rho \geq 0 .
$$

\footnotetext{
*Dipartimento di Matematica U. Dini, Università di Firenze, viale Morgagni 67/A, 50134 Firenze, Italy.

${ }^{\dagger}$ Current Address: Dipartimento di Scienze e Metodi dell'Ingegneria, Università degli Studi di Modena e Reggio Emilia, via G. Amendola, 2 - Pad. Morselli - 42100 Reggio Emilia, Italy. Email: simone.cecchini@unimore.it

${ }^{\ddagger}$ Dipartimento di Matematica U. Dini, Università di Firenze, viale Morgagni 67/A, 50134 Firenze, Italy. Email: rolando.magnanini@math.unifi.it
} 
Notice that $f$ is strictly convex and grows quadratically at infinity, thus the existence and uniqueness of a function minimizing $\mathcal{J}$ subject to a Dirichlet boundary condition is not hard to prove. However, since $f^{\prime}(0)>0, \mathcal{J}$ is not always differentiable - non-differentiability occurring when the Lebesgue measure of the set $\{x \in \Omega: \nabla u(x)=0\}$ is positive — and a standard Euler-Lagrange equation may not be available for $\mathcal{J}$, though a differential inclusion

$$
0 \in \partial \mathcal{J}(u)
$$

by means of the subdifferential $\partial \mathcal{J}$, still characterizes a minimizing $u$ (see [19] for details). A formal Euler-Lagrange equation would read

$$
\operatorname{div}\left\{f^{\prime}(|\nabla u|) \frac{\nabla u}{|\nabla u|}\right\}=0
$$

with $f^{\prime}(\rho)=\sqrt{1+\rho^{2}}$ - a clearly singular equation exactly at the critical points of $u$. Nevertheless, away from its critical points, a smooth minimizer $u$ certainly satisfies the quasilinear elliptic degenerate differential equation

$$
\operatorname{tr}\left[\mathcal{A}(\nabla u) \nabla^{2} u\right]=0
$$

(degeneration occurring, of course, at critical points), where the $N \times N$ matrix $A(p)$ has coefficients

$$
\mathcal{A}_{i j}(p)=[\alpha(|p|)-1] p_{i} p_{j}+|p|^{2} \delta_{i j}, \quad i, j=1, \ldots, N
$$

Here, $\delta_{i j}$ is the usual Kronecker's delta, while

$$
\alpha(\rho)= \begin{cases}\frac{\rho f^{\prime \prime}(\rho)}{f^{\prime}(\rho)}, & \rho>0 \\ 0, & \rho=0 .\end{cases}
$$

For $N=2$ and $f$ given by (2), Equation (4) may be formally re-written as

$$
-\left(|\nabla u|^{4}+u_{y}^{2}\right) u_{x x}+2 u_{x} u_{y} u_{x y}-\left(|\nabla u|^{4}+u_{x}^{2}\right) u_{y y}=0 .
$$

Functional (1) and equations (3), (4) show some interesting features.

Even if, as already mentioned, minimizers of $\mathcal{J}$ do not satisfy (3) in general, it is proved in [19] that they are viscosity solutions of (6). In [19], it is also shown that classical solutions of (6) exist which can not be minimizers of $\mathcal{J}$ - thus proving that the Dirichlet problem for viscosity solutions of (6) is not uniquely solvable.

Another interesting feature concerns the set of critical points of solutions of (6) - a decisive information for a good understanding of the properties of (1), (3) and (4). For $N=2$, sample solutions of (6) have their gradients which vanish on a set of positive Lebesgue measure $([18])$; also, it has been shown $([18])$ that classical solutions of (6) cannot have isolated (non-degenerate) critical points, that is their gradients either never vanish or annihilate on a continuum - a property not occurring for other well-known degenerate elliptic equations (e.g. the $p$-Laplace equation).

This phenomenon may be heuristically explained by observing that $f(\rho)$ grows only linearly near $\rho=0$, forcing the gradient of a minimizer to be "smaller 
than usual" wherever it is possible. Also, a simple inspection informs us that the operator in (3) behaves like the 1-laplacian near critical points and the ordinary laplacian for large values of $|\nabla u|$. This set of remarks make us claim that equations (3) and (4) are "more degenerate" than the $p$-Laplace equation for $1<p<\infty$ but "less degenerate" than the 1-Laplace equation, and for this reason they deserve attention.

Let us finally observe that functionals and equations with a structure similar to the one described in this subsection have been considered in the study of torsional creep problems in elasto-plastic materials ([13, [16, [22, [21]).

The aforementioned reasons motivate our interest on a more detailed analysis of such functionals and equations.

\subsection{Main results.}

We shall consider a strictly convex functional of type (1). From now on, unless differently specified, $\Omega$ will be a bounded domain in $\mathbb{R}^{N}$, while $f$ is assumed to abide to the requirements below:

$$
f \text { is strictly convex; }
$$

$$
\begin{gathered}
f \in C^{1}([0,+\infty)) \cap C_{l o c}^{2, \lambda}((0,+\infty)), 0<\lambda<1 \\
f^{\prime}(0)>0 .
\end{gathered}
$$

In order to avoid technicalities, unnecessary to the aims of our investigation, we require that the couple $(\Omega, \psi)$, where $\psi: \partial \Omega \rightarrow \mathbb{R}$ is a given continuous function, satisfies a bounded slope condition (referred to by BSC from now on; see Section 3 for details).

Under these assumptions, a classical result makes sure that the variational problem

$$
\min \{\mathcal{J}(w): w \in \operatorname{Lip}(\bar{\Omega}), w=\psi \text { on } \partial \Omega\}
$$

$\operatorname{Lip}(\bar{\Omega})$ being the space of Lipschitz continuous functions in $\bar{\Omega}$, admits a unique solution (see e.g. [11).

In Theorem 3.7, we specify sufficient conditions on $f$ that guarantee that each solution $u$ of $(8)$ is a viscosity solution of $(4)$ subject to $u=\psi$ on $\partial \Omega$. The proof of Theorem 3.7 follows the outline of the one given in [19] for the special case (2): we uniformly approximate $\mathcal{J}$ by a sequence of strictly convex differentiable functionals

$$
\mathcal{J}_{n}(u)=\int_{\Omega} f_{n}(|\nabla u|) d x
$$

whose minimizers $u_{n}$ are proven to be viscosity solutions of some relevant differential equations with coefficients that converge uniformly to those of (4). Differently from [18, the uniform convergence of the $u_{n}$ 's, needed to use the standard stability result of [17, Section 6], is easily obtained by means of the BSC.

The main result of this paper concerns the set of critical points of a solution of (8). 
Theorem 1.1. Let $u$ be a $C^{1}$ solution of (8), where $f$ satysfies (7).

Then $u$ can not have isolated critical points.

This result considerably improves the one obtained in [18 for solutions of class $C^{2}$ and settles a conjecture raised by G. Talent ${ }^{*}$ Its proof proceeds by contradiction and relies on two remarks:

(i) if a solution $u$ of $(8)$ has an isolated critical point at $z_{0} \in \Omega$, then it is a weak solution of $(3)$ in a neighborhood $\mathcal{U}$ of $z_{0}$;

(ii) even if $u$ is assumed to be only $C^{1}$ in $\mathcal{U}$, yet one can define an index $I\left(z_{0}\right)$ for the vector field $\nabla u$ in $z_{0}$ - a winding number defined on loops avoiding $z_{0}$.

Remark (i) then implies that $u$ is a classical solution of (4) in $\mathcal{U} \backslash\left\{z_{0}\right\}$ and also that there exists a (distributional) stream function $v$ for $u$ in $\mathcal{U} \backslash\left\{z_{0}\right\}$, that is a function $v$ such that

$$
\partial_{x} v=-f^{\prime}(|\nabla u|) \frac{\partial_{y} u}{|\nabla u|}, \quad \partial_{y} v=f^{\prime}(|\nabla u|) \frac{\partial_{x} u}{|\nabla u|}
$$

in $\mathcal{U} \backslash\left\{z_{0}\right\}$, in a distributional sense (stream functions will play a crucial rôle in the following sections. The reader may refer to [5] as a propaedeutic reading for what concerns Section 3 , while 2 show how stream functions have been sometimes used to infer critical-point set properties). The modulus $|\nabla v|$ of the gradient of $v$ is proven to extend continuously to $z_{0}$ and, since both $\nabla u$ and $\nabla v$ must have the same index, we infer that $\mathrm{I}\left(z_{0}\right)=0$, which entails a contradiction versus the hypothesis of $z_{0}$ being isolated. It is clear that a possible generalization of Theorem 1.1 to general dimension should rely on different arguments.

The crucial role played by the stream function $v$ in the proof of Theorem 1.1 motivates a better understanding of system 10 or its inverse

$$
\partial_{x} u=g^{\prime}(|\nabla v|) \frac{\partial_{y} v}{|\nabla v|}, \quad \partial_{y} u=-g^{\prime}(|\nabla v|) \frac{\partial_{x} v}{|\nabla v|},
$$

which can be also viewed as sorts of Cauchy-Riemann systems for $u$ and $v$. Here $g$ is the Fenchel conjugate of $f$ defined by

$$
g(r)=\sup \{\rho r-f(\rho): \rho \geq 0\}, \quad r \in[0, \infty)
$$

In other words, we want to investigate on the possibility of defining a generalized stream function $v$ associated to a solution $u$ of (8). The main difficulty with this task is that, since by Theorem $1.1 u$ may not have isolated critical points, system 10 is in general severely singular.

In this paper, we do not completely secceed in our task, but we present a few results which may help to understand the problem.

In Section 3, we show that, owing to the properties of the chosen lagrangeans $f_{n}$, the system

$$
\partial_{x} v_{n}=-f_{n}^{\prime}\left(\left|\nabla u_{n}\right|\right) \frac{\partial_{y} u_{n}}{\left|\nabla u_{n}\right|}, \quad \partial_{y} v_{n}=f_{n}^{\prime}\left(\left|\nabla u_{n}\right|\right) \frac{\partial_{x} u_{n}}{\left|\nabla u_{n}\right|}
$$

\footnotetext{
*Personal communication.
} 
can be uniquely solved by suitably normalized stream functions $v_{n}$, which are critical points of functionals,

$$
\mathcal{K}_{n}(v)=\int_{\Omega} g_{n}(|\nabla v|) d x,
$$

where $g_{n}^{\prime}=\left(f_{n}^{\prime}\right)^{-1}\left(g_{n}\right.$ is indeed the Fenchel conjugate of $f_{n}$ defined accordingly to (12)). It is evident that $u_{n}$ and $v_{n}$ also satisfy

$$
\partial_{x} u_{n}=g_{n}^{\prime}\left(\left|\nabla v_{n}\right|\right) \frac{\partial_{y} v_{n}}{\left|\nabla v_{n}\right|}, \quad \partial_{y} u_{n}=-g_{n}^{\prime}\left(\left|\nabla v_{n}\right|\right) \frac{\partial_{x} v_{n}}{\left|\nabla v_{n}\right|} .
$$

In Theorem 3.5. under appropriate assumptions on the approximating sequence $\left(f_{n}\right)_{n \in \mathbb{N}}$, we show that the sequences $\left(u_{n}\right)_{n \in \mathbb{N}}$ and $\left(v_{n}\right)_{n \in \mathbb{N}}$ contain subsequences which converge respectively to functions $u$ and $v$ satisfying (11) almost everywhere.

We are not able to prove that $u$ and $v$ also satisfy (10); however, by the same argument used in the proof of Theorem 3.7, we show that $v$ is a viscosity solution $v$ of

$$
\operatorname{tr}\left[\mathcal{B}(\nabla v) \nabla^{2} v\right]=0,
$$

where $\mathcal{B}(p)$ is a matrix whose coefficients are the uniform limits of

$$
-\left[1-\alpha_{n}\left(g_{n}^{\prime}(|p|)\right)\right] p_{i} p_{j}-|p|^{2} \alpha_{n}\left(g_{n}^{\prime}(|p|)\right) \delta_{i j} \quad i, j=1, \ldots, 2,
$$

with

$$
\alpha_{n}(\rho)= \begin{cases}\frac{\rho f_{n}^{\prime \prime}(\rho)}{f_{n}^{\prime}(\rho)}, & \rho>0, \\ \lim _{\rho \rightarrow 0^{+}} \frac{\rho f_{n}^{\prime \prime}(\rho)}{f_{n}^{\prime}(\rho)}, & \rho=0 .\end{cases}
$$

This is the content of Theorem 3.8

It is worth mentioning that the analytic form of $\mathcal{B}$ may depend upon the particular approximating sequence $\left(f_{n}\right)_{n \in \mathbb{N}}$ adopted, that is, different approximations lead to different limit equations (see Remark 3.10 for details). One of these choices leads to the following interesting equation for $v$ :

$$
-\left[1-\alpha\left(g^{\prime}(|\nabla v|)\right)\right] \Delta_{\infty} v-|\nabla v|^{2} \alpha\left(g^{\prime}(|\nabla v|)\right) \Delta v=0,
$$

where $g$ is given by (12). Notice that, for values of $|\nabla v|$ less than or equal to $f^{\prime}(0), v$ must be $\infty$-harmonic.

\section{Critical points of minimizers}

This section will be devoted to the proof of our main result (Theorem 1.1), which will be consequence of Lemma 2.1 and Theorem 2.5. which may be of independent interest.

Lemma 2.1. Let $u$ be a solution of (8) with $f$ satisfying (7). If $u \in C^{1}(\Omega)$ and the set $\{z \in \Omega:|\nabla u(z)|=0\}$ has zero Lebesgue measure, then $u$ is a weak solution of (3) in $\Omega$.

In particular, if $z_{0} \in \Omega$ is an isolated critical point for $u$, then there exists a neighborhood of $z_{0}$ in which $u$ is a weak solution of (3). 
Proof. It is easy to see that, for any test function $\varphi$ whose support is contained in $\Omega$, the derivative of $\mathcal{J}$ in the direction given by $\varphi$ may be written as

$$
\partial \mathcal{J}(u)(\varphi)=\int_{\{|\nabla u| \neq 0\}} f^{\prime}(|\nabla u|)\left\langle\frac{\nabla u}{|\nabla u|}, \nabla \varphi\right\rangle d x d y+f^{\prime}(0) \int_{\{|\nabla u|=0\}}|\nabla \varphi| d x d y .
$$

By assumption, the second addendum vanishes, while the first one amounts to

$$
\int_{\Omega} f^{\prime}(|\nabla u|)\left\langle\frac{\nabla u}{|\nabla u|}, \nabla \varphi\right\rangle d x d y .
$$

If $u$ is a solution of $(8)$, then $\partial \mathcal{J}(u)(\varphi)=0$ for every test function compactly supported in $\Omega$ and hence $u$ is a weak solution of (3) in $\Omega$.

We will next proceed to compute the index $\mathrm{I}\left(z_{0}\right)$ of an isolated critical point of a solution $u \in C^{1}(\Omega)$ of 8 . We recall that $\mathrm{I}\left(z_{0}\right)$ is defined by the formula

$$
\mathrm{I}\left(z_{0}\right)=\frac{1}{2 \pi} \int_{+\gamma} \frac{u_{x} d u_{y}-u_{y} d u_{x}}{|\nabla u|^{2}},
$$

where $+\gamma$ is any loop which wraps $z_{0}$ counterclockwise and no other critical point.

Also recall the geometric meaning of the previous definition: the index of a critical point of a $C^{1}$-regular function is defined as the topological index of the vector field $\nabla u /|\nabla u|$ at the same point and that the latter corresponds to the topological degree of the field $\nabla u /|\nabla u|$ itself, considered as a map of the unit circle in itself.

Given any $r>0$ such that $B=\overline{B\left(z_{0}, r\right)} \subset \Omega$ we set our first goal to proving that the differential form

$$
\omega=\frac{f^{\prime}(|\nabla u|)}{|\nabla u|}\left(-u_{y} d x+u_{x} d y\right),
$$

which is continuous and bounded in $B^{\prime}=B \backslash\left\{z_{0}\right\}$, may be integrated to obtain a so-called stream function (see [4) $v$ which is continuous in $B$.

Notice that (3) may be cast into the form

$$
d \omega=0, \quad \text { in } \Omega,
$$

in the sense of currents/distributions, where $d$ has to be interpreted as the boundary operator (see 24]).

Lemma 2.2. Let $u: \Omega \longrightarrow \mathbb{R}$ be a $C^{1}(\Omega)$ distributional solution to (3), and let $z_{0}$ be an isolated critical point for $u$. Then the following claims hold.

(i) the period of the 1-form $\omega$ given in (18) around $z_{0}$ is null;

(ii) there exists a function $v \in C^{1}\left(B^{\prime}\right) \cap C^{0}(B)$ such that $d v=\omega$;

(iii) moreover, $|\nabla v| \in C^{0}(B)$. 
Proof. (i) Let $B_{1}=B\left(z_{0}, r / 2\right)$. We must prove that $\int_{\partial B_{1}} \omega=0$. Indeed, we know that, for any $\varphi \in C_{0}^{\infty}(B)$ it holds:

$$
\int_{B} \omega \wedge d \varphi=0 .
$$

(This is really what 19 means.) Let $\left(\eta_{\varepsilon}\right)_{\varepsilon>0}$ be a family of radially symmetric regularizing kernels, and define $\varphi_{\varepsilon}=\eta_{\varepsilon} * \chi_{B_{1}}$, where as usual $\chi_{B_{1}}$ is the characteristic function of the set $B_{1}$. Then $d \chi_{B_{1}}=\mathcal{H}^{1}\left\llcorner\partial B_{1}\left(-\nu_{1} d x-\nu_{2} d y\right)\right.$ $\left(\nu=\left(\nu_{1}, \nu_{2}\right)\right.$ is the outer unit normal to the domain $B_{1}$, while $\llcorner$ denotes the operator of restriction of measures to subsets) and $\operatorname{supp}\left(d \varphi_{\varepsilon}\right)$ is contained in a tubular $\varepsilon$-neighborhood of $\partial B_{1}$, hence taking $\varepsilon_{0}$ small enough, $z_{0} \notin \operatorname{supp}\left(d \varphi_{\varepsilon}\right)$ and $\operatorname{supp}\left(d \varphi_{\varepsilon}\right) \subset B$ for all $\varepsilon \leq \varepsilon_{0}$ and it holds $\left(\operatorname{since} \operatorname{supp}\left(d \varphi_{\varepsilon}\right) \subset \operatorname{supp}\left(d \varphi_{\varepsilon_{0}}\right)\right)$

$$
0=\int_{B} \omega \wedge d \varphi_{\varepsilon}=\int_{\operatorname{supp}\left(d \varphi_{\varepsilon_{0}}\right)} \omega \wedge d \varphi_{\varepsilon} .
$$

The last integral tends to

$$
\int_{\operatorname{supp}\left(d \varphi_{\varepsilon_{0}}\right)} \omega \wedge d \chi_{B_{1}}=\int_{\partial B_{1}} \omega .
$$

The alleged convergence is worth an explanation: we know (e.g. see 3, Thm. 2.2]) that both $d \varphi_{\varepsilon}$ tends to $d \chi_{B}$ and the total variation $\left|d \varphi_{\varepsilon}\right|$ tends to $\left|d \chi_{B}\right|$ in the sense of measures. This is enough to prove convergence in the stronger topology dual to the space of continuous and bounded functions on $\operatorname{supp}\left(d \varphi_{\varepsilon_{0}}\right)$ (see [9, Prop. 2, pg. 38]), to which $\omega$ belongs.

(ii) Thus we can integrate $\omega$, to obtain a function $v \in C^{1}\left(B^{\prime}\right)$. We claim that $v$ can be extended continuously to $B$. In fact, since $d v=\omega$, then 10 holds and hence $\nabla v \in L^{\infty}\left(B^{\prime}\right)$; therefore $v$ is (Lipschitz and a fortiori) uniformly continuous on $B^{\prime}$ and then we can extend it continuously to the border of $B^{\prime}$, in particular to $z_{0}$. This allows us to mend the domain of definition of $v$ from the topological point of view.

(iii) From the definition of $\omega$, it turns out that $|\nabla v|=f^{\prime}(|\nabla u|)$; since $u \in$ $C^{1}(\Omega)$ and $f^{\prime}$ is continuous, $|\nabla v|$ is continuous on $B$ too.

Now we prove that the index of $z_{0}$ as a critical point of the function $u$ is zero. We recall the following proposition (see e.g. [1, Lemma 3.1]).

Theorem A. Let $w$ be a real-valued $C^{1}$ function in an open set $\Omega$ in the complex plane. Let $z_{0} \in \Omega$ be an isolated critical point of $w$.

Then, one of the following cases occurs.

(i) There exists a neighborhood $\mathcal{U}$ of $z_{0}$ such that $\left\{z \in \mathcal{U}: w(z)=w\left(z_{0}\right)\right\}$ is exactly $z_{0}$, and we have $\mathrm{I}\left(z_{0}\right)=1$.

(ii) There exists a positive integer $L$ and a neighborhood $\mathcal{V}$ of $z_{0}$ such that the level set $\left\{z \in \mathcal{V}: w(z)=w\left(z_{0}\right)\right\}$ consists of $L$ simple curves. If $L \geq 2$, each pair of such curves crosses at $z_{0}$ only. We have $\mathrm{I}\left(z_{0}\right)=1-L$.

The previous theorem let us prove the following crucial statement. 


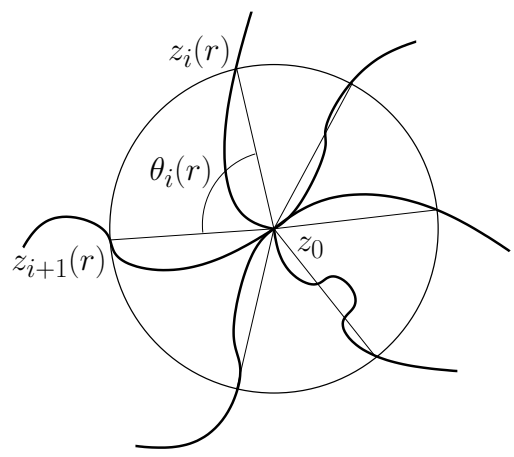

Figure 1: The level set $\left\{u=u\left(z_{0}\right)\right\}$.

Theorem 2.3. Let $u$ be a $C^{1}$ solution of (3) in the sense of distributions. Assume that $z_{0}$ is an isolated critical point for $u$.

Then the index of $z_{0}$ as a critical point is null: $\mathrm{I}\left(z_{0}\right)=0$.

Proof. In the following, we will need to recall that the level lines of $u$ correspond to the lines of steepest descent for $v$.

We apply Theorem A to the function $u$, to infer the geometry of its level set $\left\{u=u\left(z_{0}\right)\right\}$. We can exclude the case $\left\{u=u\left(z_{0}\right)\right\}=\left\{z_{0}\right\}$, for otherwise $z_{0}$ would be a local extremum for $u$ and hence for some $\varepsilon>0)$ either $\left\{u=u\left(z_{0}\right)+\varepsilon\right\}$ or $\left\{u=u\left(z_{0}\right)-\varepsilon\right\}$ would be a closed curve $\gamma$ winding around $z_{0}$ thus implying that

$$
\int_{+\gamma} \omega \neq 0 .
$$

But this would contradict Lemma 2.2 (ii).

Let $2 L$ be the number of branches of $\left\{u=u\left(z_{0}\right)\right\}$. (Caveat: in the present terminology, a branch is any arc in which any of the curves whose existence is stated by Theorem $\mathrm{A}$ is split into by $z_{0}$. Hence, when the curves are $L$, the branches are exactly $2 L$.) Denote them by $\gamma_{1} \ldots \gamma_{2 L}$; here the subscripts are assigned in the counterclockwise order of occurrence, starting from an arbitrary branch.

We preliminarly observe that each $\gamma_{i}$ is rectifiable. Indeed, let $z, z^{\prime} \in \gamma_{i} \cap B^{\prime}$; since $\gamma_{i}$ is a curve of steepest descent for $v$ and $\left|\nabla v\left(z_{0}\right)\right| \leq|\nabla v|$ on $B$, we can write that

$$
\max _{B}\left|v-v\left(z^{\prime}\right)\right| \geq v(z)-v\left(z^{\prime}\right)=\int_{0}^{l}\left|\nabla v\left(\gamma_{i}(s)\right)\right| d s \geq l\left|\nabla v\left(z_{0}\right)\right|,
$$

where $l$ is the length of the $\operatorname{arc}$ on $\gamma_{i}$ joining $z^{\prime}$ to $z$. Thus, we discover that $l$ remains bounded as $z^{\prime} \rightarrow z_{0}$, since $\left|\nabla v\left(z_{0}\right)\right|>0$.

When $r$ is small enough, each $\gamma_{i}$ crosses the circle $\left\{z:\left|z-z_{0}\right|=r\right\}$ in one point $z_{i}(r)$, for $i=1 \ldots 2 L$. Hence, setting $\theta_{i}(r)$ as the angle between the two directions $z_{i}(r)-z_{0}$ and $z_{i+1}(r)-z_{0}$ for $i=1 \ldots 2 L$ and $z_{2 L+1}(r)=z_{1}(r)$, it holds that

$$
\sum_{i=1}^{2 L} \theta_{i}(r)=2 \pi .
$$


Now consider two consecutive branches, say $\gamma_{i}, \gamma_{i+1}$; we may assume that $v$ is increasing along $\gamma_{i}$ and decreasing along $\gamma_{i+1}$, away from $z_{0}$. (It is easy to see that the case in which $v$ is increasing — or decreasing - along two consecutive branches is not consistent with the present case.) Since the two branches are rectifiable, then we can infer that

$$
\begin{aligned}
v\left(z_{i}\right) & =v\left(z_{0}\right)+\left|\nabla v\left(z_{0}\right)\right| r+o(r), \\
v\left(z_{i+1}\right) & =v\left(z_{0}\right)-\left|\nabla v\left(z_{0}\right)\right| r+o(r),
\end{aligned}
$$

as $r$ approaches 0 , where we have set for short $z_{i}=z_{i}(r), z_{i+1}=z_{i+1}(r)$.

At the same time, there exists $\xi \in\left[z_{i}, z_{i+1}\right]$ (here $\left[z_{i}, z_{i+1}\right]$ is the line segment joining $z_{i}$ to $z_{i+1}$ ) such that the following inequality holds

$$
v\left(z_{i}\right)-v\left(z_{i+1}\right) \leq|\nabla v(\xi)| \cdot\left|z_{i}-z_{i+1}\right| .
$$

Thus, we conclude

$$
|\nabla v(\xi)| \geq \frac{v\left(z_{i}\right)-v\left(z_{i+1}\right)}{\left|z_{i}-z_{i+1}\right|} \geq \frac{2 \cdot\left|\nabla v\left(z_{0}\right)\right|+o(1)}{\sqrt{2\left[1-\cos \theta_{i}\right]}} .
$$

Let $\Theta$ be any limit point of $\theta_{i}=\theta_{i}(r)$, as $r$ tends to 0 . Then we obtain (eventually by taking subsequences) that

$$
\left|\nabla v\left(z_{0}\right)\right|=\liminf _{r \rightarrow 0}|\nabla v(\xi)| \geq \frac{2}{\sqrt{2(1-\cos \Theta)}}\left|\nabla v\left(z_{0}\right)\right| \geq\left|\nabla v\left(z_{0}\right)\right| .
$$

Therefore $\Theta=\pi$.

Thus we have proved that two branches of $\left\{u=u\left(z_{0}\right)\right\}$ cannot exist such that the angle formed by the limit tangent versor to the branches is different from $\pi$. But then 20 informs that the level set $\left\{u=u\left(z_{0}\right)\right\}$ is made of no more than two branches, i.e. one curve. Hence $L=1$ and then, as stated by Theorem $\mathrm{A}$ the index of $z_{0}$ as a critical point is zero.

Lemma 2.4. Let $w$ be any $C^{2}$ function and let $t>0$ be any regular value of $|\nabla w|$.

Then

$$
\int_{+\gamma_{t}} \frac{w_{x} d w_{y}-w_{y} d w_{x}}{|\nabla w|^{2}}=\int_{+\gamma_{t}} \frac{\operatorname{det}\left(\nabla^{2} w\right)}{\left|\nabla^{2} w \nabla w\right|} d s
$$

where $\gamma_{t}=\{z: w(z)=t\}$ and $s$ denotes the arc-length.

Proof. We have

$$
\int_{+\gamma_{t}} \frac{w_{x} d w_{y}-w_{y} d w_{x}}{|\nabla w|^{2}}=\int_{+\gamma_{t}} \frac{\left(w_{x} w_{x y}-w_{y} w_{x x}\right) d x+\left(w_{x} w_{y y}-w_{y} w_{x y}\right) d y}{|\nabla w|^{2}} .
$$

Since $t$ is a regular value, $\gamma_{t}$ is made of regular curves and

$$
\left(\begin{array}{l}
d x \\
d y
\end{array}\right)=\frac{d s}{\left|\nabla^{2} w \nabla w\right|}\left(\begin{array}{r}
-w_{x} w_{x y}-w_{y} w_{y y} \\
w_{x} w_{x x}+w_{y} w_{x y}
\end{array}\right)
$$

on $\gamma_{t}$. The conclusion follows at once after simple algebraic manipulations. 
Theorem 2.5. Let $u$ be a $C^{1}$ solution in the sense of distributions of (3). Assume $f$ satisfies (7).

Then $u$ can not have isolated critical points.

Proof. Let $\Omega^{\prime}$ denote the open subset of $\Omega$ in which $|\nabla u|>0$. On any open subset $A$ of $\Omega^{\prime}$ whose closure is contained in $\Omega^{\prime},|\nabla u|$ is bounded away from zero; thus we can apply [11, Thm. 10.18] to infer that $u$ has Hölder continuous second derivatives in any such $A$ and hence in $\Omega^{\prime}$ (notice that, under our assumptions (7) on $f$, it is a standard computation to prove that $\left.u \in W^{2,2}\left(\Omega^{\prime}\right)\right)$. Also, on any such $A$, Sard's lemma in the version of 15 may be applied to $|\nabla u|^{2}$ in $A$; in particular, we have that

$$
\int_{A} \frac{\operatorname{det}\left(\nabla^{2} u\right)}{|\nabla u|} d x d y=\int_{m}^{M}\left(\int_{\gamma_{t}} \frac{\operatorname{det}\left(\nabla^{2} u\right)}{\left|\nabla^{2} u \nabla u\right|}\right) d t
$$

by the coarea formula (see [8, Thm. 1, Sec. 3.4.2]), where $m=\min _{\bar{A}}|\nabla u|$, $M=\max _{\bar{A}}|\nabla u|$.

Now $z_{0}$ is a strict minimum point for $|\nabla u|$ such that the connected component $\tilde{A}$ of $\left\{z \in \Omega^{\prime}:|\nabla u(z)|<\varepsilon_{0}\right\}$ containing $z_{0}$ is bounded by a simple closed curve. We now choose $A_{\varepsilon}=\left\{z \in \tilde{A}: \varepsilon<|\nabla u(z)|<\varepsilon_{0}\right\}$ for $0<\varepsilon<\varepsilon_{0}$ and apply 21 and Lemma 2.4 we get

$$
\int_{A_{\varepsilon}} \frac{\operatorname{det}\left(\nabla^{2} u\right)}{|\nabla u|} d x d y=\int_{\varepsilon}^{\varepsilon_{0}}\left(\int_{\gamma_{t}} \frac{u_{x} d u_{y}-u_{y} d u_{x}}{|\nabla u|^{2}}\right) d t .
$$

Formula (17) and Theorem 2.3 yield that the integrand on the right-hand side of the latter is null for almost every $t \in\left(\varepsilon, \varepsilon_{0}\right)$, hence

$$
\int_{A_{\varepsilon}} \frac{\operatorname{det}\left(\nabla^{2} u\right)}{|\nabla u|} d x d y=0
$$

Now, $\operatorname{det}\left(\nabla^{2} u\right) \leq 0$ in $A_{\varepsilon}$, because $u$ satysfies an elliptic equation; thus $\operatorname{det}\left(\nabla^{2} u\right) \equiv$ 0 in $A_{\varepsilon}$ and hence in $A_{0}$, since $\varepsilon \in\left(0, \varepsilon_{0}\right)$ is arbitrary. An application of Bernstein's inequality (e.g. see [10, Problem 12.3] or [25]) yields that

$$
\left|\nabla^{2} u\right|^{2} \leq-\operatorname{det}\left(\nabla^{2} u\right)\left[\alpha(|\nabla u|)+\alpha(|\nabla u|)^{-1}\right]
$$

and hence that $u$ is affine in $A_{0}$ and, by continuity, constant in a whole neighborhood of $z_{0}$ (recall that $\left.\left|\nabla u\left(z_{0}\right)\right|=0\right)$. This is a contradiction.

Proof of Theorem 1.1. By Lemma 2.1 u satisfies (3) in the sense of distributions. Therefore Theorem 2.5 applies.

Remark 2.6. It is easy to see that a result similar to that of Theorem 1.1 can be proved also for $C^{1}$ solutions of $(10)$ : if $(u, v)$ is a $C^{1}$ pair satisfying (10), then $u$ cannot have isoltated critical points. Indeed, in this case Theorem 2.3 holds for $v$ taken as the second component of the solution pair. 
Remark 2.7. A slight modification of the example in [18, Sec. 2.2] proves that there exist solutions of (8) whose set of critical points has zero Lebesgue measure. Indeed the function $u$ defined by

$$
u(x, y)=\left\{\begin{aligned}
1-\sqrt{\frac{1-x^{2}-y^{2}}{2}+\frac{1}{2} \sqrt{\left(1-x^{2}-y^{2}\right)^{2}+4 y^{2}}} & x \geq 0, \\
-1+\sqrt{\frac{1-x^{2}-y^{2}}{2}+\frac{1}{2} \sqrt{\left(1-x^{2}-y^{2}\right)^{2}+4 y^{2}}} & x<0,
\end{aligned}\right.
$$

is a distributional solution of class $C^{1,1}$ of $(3)$ in every ball $B(0, R), 0<R<1$, and hence solves 8 with $\psi=u_{\left.\right|_{\partial B(0, R)}}$.

\section{Stream Functions and Viscosity Approxima- tions}

The reader should refer to [17] for a definition and relevant developments concerning viscosity solutions ([14] for an account more appropriate to novices). Lemma 3.2 resumes the only non-standard property of viscosity solutions needed in the following.

Here, we shall analyse the relationships between functional (1) and equation (4); we will also set up a framework that, in certain instances, leads to the construction of a stream function associated to the unique solution of (8).

We shall assume that $f$ fulfills $(7)$ and that the ratio $\frac{\rho f^{\prime \prime}(\rho)}{f^{\prime}(\rho)}$ has finite limits as $\rho \rightarrow 0^{+}$and $\rho \rightarrow+\infty$. It is easy to show that on account of $(7 \mathrm{c})$, the first limit is zero. Thus, we adopt the definition (5) of the function $\alpha:[0,+\infty) \rightarrow[0,+\infty)$ and we assume

$$
\alpha_{\infty}:=\lim _{\rho \rightarrow+\infty} \frac{\rho f^{\prime \prime}(\rho)}{f^{\prime}(\rho)}>0 .
$$

Observe incidentally that 222 and $7 \mathrm{~b}$ imply that there exist constants $q_{1}, q_{2} \in(1,+\infty), q_{1}<q_{2}$ and $c_{1}, c_{2}>0$ such that, for $\rho \geq 0$

$$
\begin{gathered}
c_{1} \rho^{q_{1}}-c_{2} \leq f(\rho) \leq c_{1} \rho^{q_{2}}+c_{2}, \\
c_{1} \rho^{q_{1}-1}-c_{2} \leq f^{\prime}(\rho) \leq c_{1} \rho^{q_{2}-1}+c_{2} .
\end{gathered}
$$

As starters, we recall a classical result, providing a short proof of it tailored on our purposes. We will say that a couple $(\Omega, \psi)$, where $\psi: \partial \Omega \rightarrow \mathbb{R}$ is a continuous function, satisifies a bounded slope condition (BSC for short) with constant $Q>0$ if, for every $x_{0} \in \partial \Omega$ there exist two affine functions $L^{+}$and $L^{-}$such that

$$
\begin{aligned}
& L^{-} \leq \psi \leq L^{+} \text {in } \partial \Omega, \\
& L^{-}\left(x_{0}\right)=\psi\left(x_{0}\right)=L^{+}\left(x_{0}\right), \\
& \sup _{\substack{x, y \in \Omega \\
x \neq y}} \frac{\left|L^{ \pm}(x)-L^{ \pm}(y)\right|}{|x-y|} \leq Q .
\end{aligned}
$$


Proposition 3.1. Let $(\Omega, \psi)$ satisfy a BSC with constant $Q$. Assume $\left(f_{n}\right)_{n \in \mathbb{N}}$ is a sequence of strictly convex functions converging uniformly to $f$ on $[0,+\infty)$. Let $u\left(\right.$ resp. $u_{n}$ ) be the unique solution of (8) for $\mathcal{J}$ (resp. for $\mathcal{J}_{n}$ in (9p).

Then

(a) $u_{n}$ is a minimizing sequence for $\mathcal{J}$ and $\mathcal{J}_{n}\left(u_{n}\right) \rightarrow \mathcal{J}(u)$;

(b) the sequence $\left(u_{n}\right)_{n \in \mathbb{N}}$ tends to $u$ in the sup norm topology, and in the weak ${ }^{*}$ topology of $W^{1, \infty}(\Omega)$.

Proof. Since $\mathcal{J}_{n} \rightarrow \mathcal{J}$ uniformly (a) is standard. To prove (b) apply [11, Thm $1.2]$ to get the bound

$$
\left|\nabla u_{n}\right| \leq Q \quad \text { on } \bar{\Omega} .
$$

Then [7, Ch. 2, B.1] and an application of the Ascoli-Arzelà's theorem yields the desired conclusion.

In order to prove our next proposition, we need a preliminary lemma. We state it in quite a general form, since it will also be used further along, while considering the case of stream functions. The proof of Lemma 3.2 follows the lines of that of [12, Prop. 2.3] (which may also be considered as an appropriate source for a more advanced discussion of the current topic)

Lemma 3.2. Let $M: \mathbb{R}^{N} \rightarrow \mathbb{R}^{N}$ be a monotone operator such that the mapping $p \mapsto \frac{|p|^{3}}{|M(p)|} \nabla_{p} M(p)$ is continuous on $\mathbb{R}^{N}$.

Then every weak solution of

$$
\operatorname{div}(M(\nabla u))=0
$$

which is continuous and lies in $W^{1, q}(\Omega)$ for some $q>1$ is also a viscosity solution of

$$
-\frac{|\nabla u|^{3}}{|M(\nabla u)|} \operatorname{tr}\left\{\nabla_{p} M(\nabla u) \nabla^{2} u\right\}=0,
$$

where $\operatorname{tr}$ is the usual trace operator on matrices.

Proof. As usual for the viscosity setting, the proof splits into two steps: first prove that $u$ is a subsolution, then that $u$ is a supersolution. The two steps are nearly identical, thus we only go through the first one.

Assume by contradiction, that there exist $\hat{x} \in \Omega$ and $\varphi \in C^{2}(\Omega)$ satisfying

$$
\begin{gathered}
u(\hat{x})=\varphi(\hat{x}), \quad \varphi(x)>u(x), x \in \Omega \backslash\{\hat{x}\}, \\
-\frac{|\nabla \varphi(\hat{x})|^{3}}{|M(\nabla \varphi(\hat{x}))|} \operatorname{tr}\left\{\nabla_{p} M(\nabla \varphi(\hat{x})) \nabla^{2} \varphi(\hat{x})\right\}>0 .
\end{gathered}
$$

By our assumptions on $M$ and $\varphi$, the last inequality implies that $|\nabla \varphi(\hat{x})|>0$. Hence, by continuity, we can find numbers $\theta, r, t>0$ with $\theta, r>t$ such that

$$
\begin{gathered}
\overline{B_{r}} \subset \Omega, \quad \min _{B_{t}}(u-\varphi) \geq-\theta, \quad \max _{\partial B_{r}}(u-\varphi) \leq-3 \theta, \\
-\operatorname{tr}\left\{\nabla_{p} M(\nabla \varphi(\hat{x})) \nabla^{2} \varphi(\hat{x})\right\}>2 \theta, \quad-\operatorname{tr}\left\{\nabla_{p} M(\nabla \varphi) \nabla^{2} \varphi\right\}>\theta \text { on } B_{r}
\end{gathered}
$$


(here $B_{r}$ denotes the ball of radius $r$ centered at $\hat{x}$ ).

Given a non-negative $\psi \in W_{0}^{1, q}(\Omega)$, we integrate by parts on $B_{r}$ the last inequality and obtain

$$
\begin{gathered}
\int_{B_{r}}\langle M(\nabla \varphi(x)), \nabla \psi(x)\rangle= \\
\int_{B_{r}}-\operatorname{tr}\left(\nabla M(\nabla \varphi(x)) \nabla^{2} \varphi(x)\right) \cdot \psi(x) \geq \int_{B_{r}} \theta \cdot \psi(x) .
\end{gathered}
$$

Now we choose

$$
\psi(x)= \begin{cases}(u(x)-\varphi(x)+2 \theta)^{+} & \text {for any } x \in B_{r} \\ 0 & \text { elsewhere }\end{cases}
$$

then $\psi \in W_{0}^{1, q}(\Omega)$ and

$$
\nabla \psi(x)= \begin{cases}\nabla u(x)-\nabla \varphi(x) & \text { for any } x \in B_{r} \cap\{u-\varphi>2 \theta\}, \\ 0 & \text { elsewhere }\end{cases}
$$

(see e.g. [11, (3.8) pg. 86]). Thus, by (28), we have

$$
\int_{B_{r} \cap\{u-\varphi>2 \theta\}}\langle M(\nabla \varphi), \nabla u-\nabla \varphi\rangle \geq \int_{B_{r} \cap\{u-\varphi>2 \theta\}} \theta(u-\varphi+2 \theta) \geq \int_{B_{t}} \theta^{2},
$$

while, since $u$ is a weak solution of 25, it holds that

$$
\int_{B_{r} \cap\{u-\varphi>2 \theta\}}\langle M(\nabla u), \nabla u-\nabla \varphi\rangle=0 .
$$

By subtracting the latter equation to the earlier one we get a contradiction:

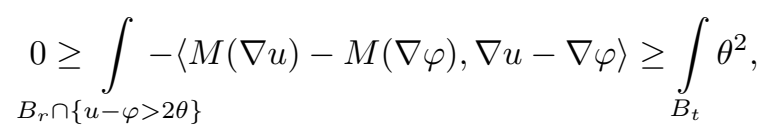

(here the first inequality follows from the monotonicity of $M$ ).

We now introduce some further assumptions on the approximating sequence $\left(f_{n}\right)_{n \in \mathbb{N}}$ considered in Proposition 3.1 and prove a couple of preliminary results.

Theorem 3.3. Assume that the $f_{n}$ 's satisfy $7 \mathrm{a}-7 \mathrm{~b}$ and

$$
f_{n}^{\prime}(0)=0, n \in \mathbb{N},
$$

and let $u_{n}$ be the solution of problem (8) for $\mathcal{J}_{n}$ in (9).

Then $u_{n}$ is a viscosity solution in $\Omega$ of

$$
-\left[\alpha_{n}(|\nabla u|)-1\right] \Delta_{\infty} u-|\nabla u|^{2} \Delta u=0,
$$

where $\alpha_{n}$ is given by (16). 
Proof. It is easy to see that - owing to $(29)$ - the Euler-Lagrange equation for the functional $(9)$ is

$$
\operatorname{div}\left(A_{n}(\nabla u)\right)=0,
$$

where $A_{n}: \mathbb{R}^{N} \rightarrow \mathbb{R}^{N}$ is the monotone operator defined by

$$
A_{n}(p)= \begin{cases}f_{n}^{\prime}(|p|) \frac{p}{|p|} & p \neq 0 \\ 0 & p=0 .\end{cases}
$$

(See also Lemma 2.1) Then apply Lemma 3.2, with $M=A_{n}$.

When $N=2$ (and $\Omega$ is simply connected), we can always define a stream function for each $u_{n}$.

Theorem 3.4. Let $\Omega \subset \mathbb{R}^{2}$ be simply connected and let the assumptions of Theorem 3.3 be in force.

Then the following assertions hold:

(i) for every $n \in \mathbb{N}$ there exists a unique Lipschitz continuous solution in the sense of distributions $v_{n}$ of the system

$$
\partial_{x} v_{n}=-f_{n}^{\prime}\left(\left|\nabla u_{n}\right|\right) \frac{\partial_{y} u_{n}}{\left|\nabla u_{n}\right|}, \quad \partial_{y} v_{n}=f_{n}^{\prime}\left(\left|\nabla u_{n}\right|\right) \frac{\partial_{x} u_{n}}{\left|\nabla u_{n}\right|},
$$

such that

$$
\int_{\Omega} v_{n}=0
$$

(ii) if $\frac{\rho f_{n}^{\prime \prime}(\rho)}{f_{n}^{\prime}(\rho)}$ converges to a positive constant as $\rho \rightarrow 0^{+}$, then $v_{n}$ is a viscosity solution of

$$
-\left[1-\alpha_{n}\left(g_{n}^{\prime}(|\nabla v|)\right)\right] \Delta_{\infty} v-|\nabla v|^{2} \alpha_{n}\left(g_{n}^{\prime}(|\nabla v|)\right) \Delta v=0,
$$

in $\Omega$, where $\alpha_{n}$ is given by (16) and $g_{n}^{\prime}$ is the inverse function of $f_{n}^{\prime}$.

Proof. (i) As a minimizer of the differentiable functional $\mathcal{J}_{n}, u_{n}$ is a weak solution of the corresponding Euler-Lagrange equation. The latter statement corresponds to saying that for any $\varphi \in W_{0}^{1, q}(\Omega)$ for $q \geq 2$, it holds that

$$
\int_{\Omega}\left\langle A_{n}\left(\nabla u_{n}(x)\right), \nabla \varphi(x)\right\rangle d x=0,
$$

where $A_{n}$ is defined in (32). The previous equation may be interpreted as the following differential form

$$
\omega_{n}= \begin{cases}\frac{f_{n}^{\prime}\left(\left|\nabla u_{n}\right|\right)}{\left|\nabla u_{n}\right|}\left(-\partial_{y} u_{n} d x+\partial_{x} u_{n} d y\right) & \left|\nabla u_{n}\right| \neq 0, \\ 0 & \text { elsewhere }\end{cases}
$$

being closed, as a form belonging to $L^{q}(\Omega)$, for $q \geq 2$.

We required the domain $\Omega$ to be simply connected. Thus (see 23, Lemma 
3.2.1]) we can integrate $\omega_{n}$ to obtain a function $v_{n} \in W^{1, q}(\Omega)$ such that (33) holds. The function $v_{n}$ is not completely defined by the condition (33): we are left with the choice of a constant to add. We choose such costant so that 34 holds.

We observe that since (24) holds and $\left|\nabla v_{n}\right|=f_{n}^{\prime}\left(\left|\nabla u_{n}\right|\right)$, we can conclude

$$
\left|\nabla v_{n}\right| \leq f_{n}^{\prime}(Q) \text { in } \Omega \text {. }
$$

(ii) Since (i) holds, $v_{n}$ is a weak solution of

$$
\operatorname{div}\left(B_{n}(\nabla v)\right)=0
$$

where $B_{n}(p)$ is the monotone operator

$$
B_{n}(p)= \begin{cases}g_{n}^{\prime}(|p|) \frac{p}{|p|} & p \neq 0 \\ 0 & p=0\end{cases}
$$

which happens to be the inverse of $A_{n}$.

Then, owing to the stated assumptions on $\alpha_{n}$, the proof follows the lines of that of Lemma 3.2 , by observing that, due to the fact that $g_{n}^{\prime}$ is the inverse of $f_{n}^{\prime}$, then

$$
\frac{r g_{n}^{\prime \prime}(r)}{g_{n}^{\prime}(r)}=\frac{1}{\alpha_{n}\left(g_{n}^{\prime}(r)\right)} .
$$

Now, we want to take the limit in $(33)$.

Theorem 3.5. Let (23) be in force and assume that $\left(f_{n}\right)_{n \in \mathbb{N}}$ is a sequence of strictly convex functions converging uniformly to $f$ on $[0,+\infty)$.

Let $u\left(\right.$ resp. $\left.u_{n}\right)$ be the unique solution of $(23)-(8)$ for $\mathcal{J}$ (resp. for $\mathcal{J}_{n}$ in (9)) and let $v_{n}$ be defined as in Theorem 3.4. Also, assume that the gradients $\nabla u_{n}$ converge to $\nabla u$ almost everywhere in $\Omega$.

Then $\left(v_{n}\right)_{n \in \mathbb{N}}$ contains a subsequence which converges uniformly on $\bar{\Omega}$ to a function $v \in W^{1, \infty}(\Omega)$ and the pair $(u, v)$ satisfies the system 11 almost everywhere in $\Omega$.

Proof. Consider the functionals

$$
\begin{aligned}
& \mathcal{F}(u, v)=\int_{\Omega}\left\{f(|\nabla u|)+g(|\nabla v|)-\left(u_{x} v_{y}-u_{y} v_{x}\right)\right\} d x d y, \\
& \mathcal{F}_{n}(u, v)=\int_{\Omega}\left\{f_{n}(|\nabla u|)+g_{n}(|\nabla v|)-\left(u_{x} v_{y}-u_{y} v_{x}\right)\right\} d x d y .
\end{aligned}
$$

From $(33)$ it is clear that $\mathcal{F}_{n}\left(u_{n}, v_{n}\right)=0$.

Now, observe that, by $(36)$ and the uniform convergence of the $g_{n}$ 's, the gradients of the $v_{n}$ 's are uniformly bounded and, since (34) holds for every $n \in \mathbb{N}$, the $v_{n}$ 's satisfy on $\bar{\Omega}$ the assumptions of Ascoli-Arzelà's theorem. Thus, $\left(v_{n}\right)_{n \in \mathbb{N}}$ contains a subsequence (that we will still denote by $\left(v_{n}\right)_{n \in \mathbb{N}}$ ) which converges uniformly on $\bar{\Omega}$ to a function $v \in W^{1, \infty}(\Omega)$ and, by the boundedness 
of $\left(v_{n}\right)_{n \in \mathbb{N}}$ in $W^{1, \infty}(\Omega)$, we can always assume that $\left(v_{n}\right)_{n \in \mathbb{N}}$ weakly converges to $v$ in any $W^{1, p}(\Omega), p>1$. The latter property implies that

$$
\left.\left.\lim _{n \rightarrow \infty} \int_{\Omega}\left(\partial_{x} u_{n} \partial_{y} v_{n}-\partial_{y} u_{n} \partial_{x} v_{n}\right)\right\} d x d y=\int_{\Omega}\left(u_{x} v_{y}-u_{y} v_{x}\right)\right\} d x d y
$$

- since the gradients $\nabla u_{n}$ are bounded and are assumed to converge a.e. to $\nabla u$ - and also that

$$
\liminf _{n \rightarrow \infty} \int_{\Omega} g_{n}\left(\left|\nabla v_{n}\right|\right) d x d y \geq \int_{\Omega} g(|\nabla v|) d x d y
$$

by the uniform convergence of $g_{n}$ to $g$ and the convexity of $g$. Therefore, we can infer that

$$
\mathcal{F}(u, v) \leq \liminf _{n \rightarrow \infty} \mathcal{F}_{n}\left(u_{n}, v_{n}\right)=0 .
$$

On the other hand, by the very definition 12 of $g$, the following inequalities hold almost everywhere in $\Omega$ :

$$
f(|\nabla u|)+g(|\nabla v|)-\left(u_{x} v_{y}-u_{y} v_{x}\right) \geq|\nabla u||\nabla v|-\left(u_{x} v_{y}-u_{y} v_{x}\right) \geq 0
$$

thus, $\mathcal{F}(u, v)=0$ and hence

$$
f(|\nabla u|)+g(|\nabla v|)-\left(u_{x} v_{y}-u_{y} v_{x}\right)=|\nabla u||\nabla v|-\left(u_{x} v_{y}-u_{y} v_{x}\right)=0
$$

almost everywhere in $\Omega$. These couple of equalities then yield 11 at once.

Remark 3.6. The a.e. convergence of the gradients $\nabla u_{n}$ assumed in Theorem 3.5 can be obtained at least in two fashions.

The former consists in applying either [6, Thm. 1] or [7, Thm. 2, pg. 21] (by possibly restricting our assumptions on $f$ ).

The latter consists in an adaptation of the arguments used in [19]: since $u_{n}$ is a solution of (30), it also satisfies the Bernstein's inequality

$$
\left|\nabla^{2} u_{n}\right|^{2} \leq-\operatorname{det}\left(\nabla^{2} u_{n}\right)\left[\alpha_{n}\left(\left|\nabla u_{n}\right|\right)+\alpha_{n}\left(\left|\nabla u_{n}\right|\right)^{-1}\right],
$$

which, by the properties of the coefficients $\alpha_{n}$, implies the bound

$$
\int_{\Omega} \alpha\left(\left|\nabla u_{n}\right|\right)\left|\nabla^{2} u_{n}\right|^{2} d x d y \leq C_{K}
$$

where $K$ is any compact subset of $\Omega$ and $C_{K}$ is a constant depending on $K$.

This last inequality provides the expected compactness of the sequence $\left(u_{n}\right)_{n \in \mathbb{N}}$.

We conclude this section by showing that the functions $u$ and $v$ determined in Theorem 3.5 are solutions of second-order degenerate elliptic equations.

Theorem 3.7. Assume the BSC (23) is in force and let $f$ satisfy (7) and (22).

Then the solution $u$ of problem (8) is a viscosity solution of

$$
-[\alpha(|\nabla u|)-1] \Delta_{\infty} u-|\nabla u|^{2} \Delta u=0 \quad \text { in } \Omega,
$$

where $\alpha$ is given by (5). 
Proof. We can always approximate $f$ by a sequence of lagrangeans $f_{n}$ such that:

(a) the $f_{n}$ 's converge to $f$ uniformly on $[0,+\infty)$;

(b) the functions $\alpha_{n}(|p|) p_{i} p_{j}$, with $\alpha_{n}$ given by $(16)$, converge to $\alpha(|p|) p_{i} p_{j}$, $i, j=1 \ldots N$, uniformly on any compact subset of $\mathbb{R}^{N}$ (see also Remark 3.10 .

By Proposition 3.1, the solutions $u_{n}$ of the minimum problem (8) rephrased in terms of $\mathcal{J}_{n}$ converge to $u$ uniformly in $\bar{\Omega}$. The conclusion then follows from [17, Lemma 6.1].

Theorem 3.8. Let $f$ satisfy (7) and (22) and suppose $f$ is approximated, uniformly on $[0, \infty)$, by a sequence of functions $f_{n}$ which obey (7a), (7b) and (29).

Assume that the functions $\alpha_{n}$ satisfy the assumptions of Theorem 3.4 and that $\alpha_{n} \circ g_{n}^{\prime}$ converge to a function $\beta$ uniformly on the compact subsets of $[0,+\infty)$.

Then the sequence $\left(v_{n}\right)_{n \in \mathbb{N}}$ contains a subsequence that converges uniformly in $\Omega$ to a function a viscosity solution $v$ of

$$
-[1-\beta(|\nabla v|)] \Delta_{\infty} v-|\nabla v|^{2} \beta(|\nabla v|) \Delta v=0 \quad \text { in } \Omega .
$$

Proof. By Theorem 3.4 each $v_{n}$ satisfies (35); (36) and the properties of the $f_{n}$ 's imply that the sequence $\left(v_{n}\right)_{n \in \mathbb{N}}$ satisfies the assumptions of Ascoli-Arzelà's theorem. Therefore the conclusion follows again by applying [17, Lemma 6.1] to any uniformly converging subsequence of $\left(v_{n}\right)_{n \in \mathbb{N}}$.

Corollary 3.9. If the coefficients $\alpha_{n}$ also converge to $\alpha$ uniformly on every compact subset of $[0,+\infty)$, then the function $v$ of Theorem 3.8 is a viscosity solution of

$$
-\left[1-\alpha\left(g^{\prime}(|\nabla v|)\right)\right] \Delta_{\infty} v-|\nabla v|^{2} \alpha\left(g^{\prime}(|\nabla v|)\right) \Delta v=0 \quad \text { in } \Omega,
$$

where $g(r)=\sup \{r \rho-f(\rho): \rho \geq 0\}$.

Proof. It is enough to compute the function $\beta$. Since $g_{n}(r)$ always converge to $g^{\prime}(r)$, we obtain that $\beta(r)=\alpha\left(g^{\prime}(r)\right)$ at once.

Remark 3.10. Sequences of $\left(f_{n}\right)_{n \in \mathbb{N}}$ that satisfy the assumptions mentioned in the statement of Theorem 3.8 can be constructed in various fashions. A convenient way is to modify $f$ only in a neighborhood of $\rho=0$.

Here we give two examples; we set $f_{n}^{\prime}(\rho)=\sigma_{n}(\rho) f^{\prime}(\rho)$, with $\sigma_{n}(\rho) \equiv 1$ for $\rho \geq 1$, while

(a) $\sigma_{n}^{a}(\rho)=1-\left(1-\rho^{\frac{1}{n}}\right)^{n}$ for $\rho \in[0,1]$;

(b) $\sigma_{n}^{b}(\rho)=1-\left(1-\rho^{s}\right)^{n}$ for $\rho \in[0,1]$

where $s>0$.

With the obvious notations it easy to see that the $\alpha_{n}^{a}$ 's converge uniformly to $\alpha$ on $[0,+\infty)$, so that Corollary 3.9 applies. In particular, $\alpha \circ g^{\prime} \equiv 0$ on $\left[0, f^{\prime}(0)\right]$ so that, for $|\nabla v| \leq f^{\prime}(0),(39)$ reads thus: $\Delta_{\infty} v=0$. 
The $\alpha_{n}^{b}$ 's instead converge uniformly in the compact subsets of $(0, \infty)$ but not on those of $[0, \infty)$. Straightforward computations show that the function $v$ of Theorem 3.8 satisfies 38 , where

$$
\beta(r)= \begin{cases}-s \frac{f^{\prime}(0)-r}{r} \log \left[1-r / f^{\prime}(0)\right] & 0 \leq r \leq f^{\prime}(0), \\ \alpha\left(g^{\prime}(r)\right) & r>f^{\prime}(0) .\end{cases}
$$

Acknoledgements. The authors are indebted with Prof. G. Talenti for the many helpful discussions and, in particular, for inspiring the use of functional $\mathcal{F}(u, v)$ in Theorem 3.5

\section{References}

[1] G. Alessandrini and R. Magnanini. The index of isolated critical points and solutions of elliptic equations in the plane. Ann. Scuola Norm. Sup. Pisa Cl. Sci. (4), 19(4):567-589, 1992.

[2] G. Alessandrini and R. Magnanini. Elliptic equations in divergence form, geometric critical points of solutions, and Stekloff eigenfunctions. SIAM J. Math. Anal., 25(5):1259-1268, 1994.

[3] L. Ambrosio, N. Fusco, and D. Pallara. Functions of bounded variation and free discontinuity problems. Oxford Mathematical Monographs. The Clarendon Press Oxford University Press, New York, 2000.

[4] G. Aronsson. On certain p-harmonic functions in the plane. Manuscripta Math., 61(1):79-101, 1988.

[5] G. Aronsson and P. Lindqvist. On $p$-harmonic functions in the plane and their stream functions. J. Differential Equations, 74(1):157-178, 1988.

[6] L. C. Evans and R. F. Gariepy. Some remarks concerning quasiconvexity and strong convergence. Proc. Roy. Soc. Edinburgh Sect. A, 106(1-2):53-61, 1987.

[7] L.C. Evans. Weak convergence methods for nonlinear partial differential equations, volume 74 of CBMS Regional Conference Series in Mathematics. Published for the Conference Board of the Mathematical Sciences, Washington, DC, 1990.

[8] L.C. Evans and R.F. Gariepy. Measure theory and fine properties of functions. Studies in Advanced Mathematics. CRC Press, Boca Raton, FL, 1992.

[9] M. Giaquinta, G. Modica, and J. Souček. Cartesian currents in the calculus of variations. I, volume 37 of Ergebnisse der Mathematik und ihrer Grenzgebiete. 3. Folge. A Series of Modern Surveys in Mathematics [Results in Mathematics and Related Areas. 3rd Series. A Series of Modern Surveys in Mathematics]. Springer-Verlag, Berlin, 1998. Cartesian currents.

[10] D. Gilbarg and N.S. Trudinger. Elliptic partial differential equations of second order. Classics in Mathematics. Springer-Verlag, Berlin, 2001. Reprint of the 1998 edition. 
[11] E. Giusti. Direct methods in the calculus of variations. World Scientific Publishing Co. Inc., River Edge, NJ, 2003.

[12] T. Ishibashi and S. Koike. On fully nonlinear PDEs derived from variational problems of $L^{p}$ norms. SIAM J. Math. Anal., 33(3):545-569 (electronic), 2001.

[13] B. Kawohl. On a family of torsional creep problems. J. Reine Angew. Math., 410:1-22, 1990.

[14] S. Koike. A beginner's guide to the theory of viscosity solutions, volume 13 of MSJ Memoirs. Mathematical Society of Japan, Tokyo, 2004.

[15] M.V. Korobkov. On an analogue of Sard's theorem for $C^{1}$-smooth functions of two variables. Sibirsk. Mat. Zh., 47(5):1083-1091, 2006.

[16] A. Langenbach. Verallgemeinerte und exakte Lösungen des Problems der elastisch-plastischen Torsion von Stäben. Math. Nachr., 28:219-234, $1964 / 1965$.

[17] H. Ishii M. G. Crandall and P.L. Lions. User's guide to viscosity solutions of second order partial differential equations. Bull. Amer. Math. Soc. (N.S.), $27(1): 1-67,1992$

[18] R. Magnanini and G. Talenti. On complex-valued solutions to a 2-D eikonal equation. I. Qualitative properties. In Nonlinear partial differential equations (Evanston, IL, 1998), volume 238 of Contemp. Math., pages 203-229. Amer. Math. Soc., Providence, RI, 1999.

[19] R. Magnanini and G. Talenti. On complex-valued solutions to a twodimensional eikonal equation. II. Existence theorems. SIAM J. Math. Anal., 34(4):805-835 (electronic), 2003.

[20] R. Magnanini and G. Talenti. On complex-valued solutions to a 2D eikonal equation. III. Analysis of a Bäcklund transformation. Appl. Anal., 85(13):249-276, 2006.

[21] L.E. Payne and G.A. Philippin. Some applications of the maximum principle in the problem of torsional creep. SIAM J. Appl. Math., 33(3):446-455, 1977.

[22] G.A. Philippin. A minimum principle for the problem of torsional creep. J. Math. Anal. Appl., 68(2):526-535, 1979.

[23] G. Schwarz. Hodge decomposition - a method for solving boundary value problems, volume 1607 of Lecture Notes in Mathematics. Springer-Verlag, Berlin, 1995.

[24] L. Simon. Lectures on geometric measure theory, volume 3 of Proceedings of the Centre for Mathematical Analysis, Australian National University. Australian National University Centre for Mathematical Analysis, Canberra, 1983.

[25] G. Talenti. Equazioni lineari ellittiche in due variabili. Matematiche (Catania), 21:339-376, 1966. 